\title{
Estimativas da trajetória genética do crescimento de codornas de corte utilizando modelos de regressão aleatória
}

[Genetic trajectory estimates of meat type quail lines using random regression models]

\author{
N.J.L. Dionello ${ }^{1,4}$, G.S.S. Correa $a^{2,4}$, M.A. Silva $a^{2,4}$, A.B. Corrêa ${ }^{3}$, G.G. Santos ${ }^{3}$ \\ ${ }^{1}$ Faculdade de Agronomia Eliseu Maciel - UFPel \\ Campus Universitário \\ 96010-900 - Pelotas, RS \\ ${ }^{2}$ Escola de Veterinária - UFMG - Belo Horizonte, MG \\ ${ }^{3}$ Aluno de pós-graduação - EV-UFMG - Belo Horizonte, MG \\ ${ }^{4}$ Bolsista do $\mathrm{CNPq}$ \\ RESUMO
}

\begin{abstract}
Avaliou-se a trajetória genética do crescimento de codornas de corte (pesos corporais nos dias 1, 7, 14, 21, 28, 35 e 42 de idade) de dois grupos genéticos, por modelos de regressão aleatória, sob modelo animal, obtendo-se estimativas de parâmetros genéticos visando definir a idade que resultaria em maior resposta à seleção. Foram pesadas 2.432 e 2.478 codornas, respectivamente, dos grupos genéticos EV1 e EV2, obtidas em três gerações de seleção. Os parâmetros genéticos foram estimados utilizando-se a subrotina Dxmrr do DFReml. As estimativas de herdabilidade dos pesos corporais variaram, respectivamente, de 0,01 a 0,50, para o grupo genético EV1, e de 0,01 a 0,10, para o EV2, sendo as maiores estimativas obtidas para peso corporal no $42^{\circ}$ dia de idade, em ambos os grupos genéticos. As correlações genéticas entre o peso a um dia e os demais pesos corporais, em ambos os grupos, foram negativas, e as correlações de ambiente permanente, de modo geral, foram maiores para EV2. Pode-se concluir que, para ambos os grupos, a seleção é mais eficiente se realizada no $42^{0}$ dia de idade, porém respostas poderiam ser obtidas se as codornas fossem selecionadas em idades mais jovens para o grupo EV1.
\end{abstract}

Palavras-chave: codorna de corte, trajetória genética, herdabilidade, correlação genética, correlação de posto

\begin{abstract}
Growth genetic trajectories of two quail meat type lines for body weights at hatch and 7, 14, 21, 28, 35, and 42 days of age were evaluated using random regression model, under animal model analysis. Genetic parameters estimates were obtained to establish the most adequate age for efficient selection. Body weights of 2,432 and 2,478 quails from EV1 and EV2 genetic lines, respectively, from three generations of selection were recorded. Genetic parameter estimates were obtained using Dxmrr subroutine of the DFReml software. Heritability estimates ranged from .01 to .50 for EV1 and from 01 to .10 for EV2. For both genetic lines the highest heritability estimates were obtained for body weight at 42 days of age and genetic correlations between body weight at hatch and body weights recorded in other ages were all negatives and permanent environment correlations were higher for EV2 genetic line. The results suggest that selection to increase body weight for both genetic lines is more efficient when undertaken at 42 days of age, but reasonable responses to selection can also be obtained in younger ages for EV1 genetic line.
\end{abstract}

Keywords: Meat type quail, genetic trajectory, heritability, genetic correlation, rank correlation

Recebido em 5 de janeiro de 2007

Aceito em 10 de março de 2008

E-mail: dionello@ufpel.edu.br 


\section{INTRODUÇÃO}

O Brasil não dispõe de material genético de codornas, tanto para a produção de ovos como para a produção de carne, e ambos os sistemas de produção ficam na dependência de matrizes importadas.

As codornas de corte apresentam maior porte e são mais indicadas para a produção de carne por obterem maiores pesos na mesma idade de abate do que as codornas japonesas, que têm sido utilizadas também para a produção de carne (Corrêa et al.,2006; Corrêa et al., 2007abcd).

Para se obter materiais genéticos de qualidade, são necessários programas de melhoramento bem fundamentados, embasados em parâmetros genéticos acurados e precisos.

Os programas de melhoramento animal necessitam também de constante acompanhamento das características de importância econômica de cada linhagem.

A partir de informações de parâmetros genéticos, são estabelecidos critérios de seleção para garantir a renovação dos plantéis com animais de potencial genético superior, geração após geração.

Os ciclos envolvidos na produção de linhagens, quer o referente à seleção das linhas puras embasados nas variações genéticas aditivas, quer o da seleção dos cruzamentos entre essas linhas puras nos quais as variações epistáticas ou de sobredominância tornam-se importantes, são longos e exigem recursos ao lado da continuidade dos programas.

O uso de modelos de regressão aleatória tem sido proposto como alternativa para modelar características de crescimento, que são medidas repetidas na vida dos animais, sendo consideradas como dados longitudinais.

Medidas repetidas de um mesmo indivíduo podem ser analisadas sob diferentes aspectos metodológicos. As abordagens mais utilizadas são os modelos de repetibilidade, que consideram todas as medidas como sendo o mesmo caráter (Johnston e Bunter, 1996; Pereira et al., 2000), ou os modelos de multicaracterísticas, que consideram cada medida como um caráter diferente (Johnston e Bunter, 1996; Gressler et al., 2000).

Em contraste com os modelos multicaracterísticas, que fornecem predições para determinados pontos ou idades, os modelos de regressão aleatória permitem a predição de valores genéticos para a curva de crescimento como um todo, para qualquer ponto desejado na escala de tempo utilizada e para funções da curva. Com isto, permitem melhor utilização dos dados, já que todas as medidas do animal e de seus parentes são usadas para avaliação genética com potencial aumento da acurácia de seleção.

Os modelos de regressão aleatória (MRA) têm sido propostos como alternativa na avaliação genética de dados longitudinais, pois suas funções de covariância resultantes são as mais apropriadas para a análise. Segundo El Faro e Albuquerque (2003), os MRA podem ser chamados também de modelos de dimensão infinita, uma vez que existem medidas infinitas por indivíduo, e tais medidas são mais correlacionadas, quanto mais próximas elas forem.

Os MRA acomodam registros repetidos para caracteres que mudam gradualmente ao longo do tempo, não requerendo pressuposições quanto à constância das variâncias e correlações (Meyer, 2000), e têm sido usados no melhoramento animal para modelar registros diários de produção de leite durante a lactação de bovinos leiteiros, assim como no crescimento corporal em bovinos de corte (Albuquerque e Meyer, 2001). Igualmente, os MRA permitem ajustar uma trajetória aleatória para cada indivíduo como desvios de uma trajetória média da população, descrevendo os desvios genéticos a partir de regressões fixas permitindo que cada animal tenha uma forma diferente da trajetória de seus desempenhos em termos genéticos (Meyer, 1998b).

Assim, o objetivo neste trabalho foi avaliar as características de crescimento de codornas de corte (pesos corporais nos dias 1, 7, 14, 21, 28, 35 e 42 de idade) de duas linhagens por meio de modelos de regressão aleatória, sob modelo animal, visando obter estimativas de parâmetros genéticos mais confiáveis dos pesos corporais ao longo da curva de crescimento e, 
conseqüentemente, maior eficiência na seleção das aves.

\section{MATERIAL E MÉTODOS}

Foram analisadas informações de pesos corporais registrados durante o período de crescimento de 2.432 e 2.472 codornas de corte, respectivamente, dos grupos genéticos EV1 e EV2, provenientes de três gerações sucessivas de seleção pelo peso corporal aos 42 dias de idade, dentro do programa de melhoramento genético de codornas, desenvolvido na Escola de Veterinária da Universidade Federal de Minas Gerais. Para controle de pedigree, machos e fêmeas foram alojados individualmente em gaiolas, sendo a reprodução realizada levando-se o macho às fêmeas, numa proporção de um macho para duas fêmeas. Para o controle de pedigree, os ovos foram coletados, identificados por pai e mãe por meio de adesivos numerados, e incubados.

No $17^{\circ}$ dia, os ovos foram transferidos para bandejas de eclosão devidamente divididas em células que permitiram as separações das famílias de irmãos completos.
Ao nascimento, cada uma das codorninhas foi pesada e identificada por intermédio de anilha especialmente confeccionada na UFMG, a partir de canudinhos usados para consumo de refrigerantes, com dimensão de quatro $\mathrm{mm}$, e numerados seqüencialmente, de tal forma a permitir o controle de pedigree. As anilhas, por não serem expansíveis, foram trocadas por anilhas maiores e importadas, a partir do $7^{\circ}$ dia idade. A característica principal avaliada foi $o$ peso corporal, que foi registrado ao nascimento e aos sete, 14, 21, 28, 35 e 42 dias de vida das codornas, sendo a seleção das codornas para a reprodução da próxima geração realizada no $42^{\circ}$ dia de idade.

O período de coleta de ovos para incubação foi após a $17^{\mathrm{a}}$ semana de idade das codornas nas gerações um e três e na sétima semana para a geração dois.

O número de fêmeas utilizadas da primeira à terceira geração e o número de aves pesadas até o final do período, para os dois grupos genéticos, são apresentados na Tab1.

Tabela 1. Número de fêmeas utilizadas e de codornas pesadas até o final do período para os dois grupos genéticos, em cada geração

\begin{tabular}{|c|c|c|c|c|}
\hline \multirow{2}{*}{ Geração } & \multicolumn{2}{|c|}{ Grupo genético EV1 } & \multicolumn{2}{|c|}{ Grupo genético EV2 } \\
\hline & Mães & Filhos & Mães & Filhos \\
\hline Primeira & 148 & 524 & 119 & 460 \\
\hline Segunda & 207 & 460 & 183 & 401 \\
\hline Terceira & 177 & 1.448 & 164 & 1.617 \\
\hline Total & & 2.432 & & 2.478 \\
\hline
\end{tabular}

O modelo utilizado na análise de regressão aleatória da trajetória genética do crescimento das codornas, sob modelo animal foi:

$$
\begin{aligned}
& \mathrm{y}_{\mathrm{ijkm}}=\mathrm{S}_{\mathrm{j}}+\mathrm{GC}_{\mathrm{k}}+\Sigma \mathrm{Z}_{\mathrm{dm}} \mathrm{a}_{\mathrm{di}}+\Sigma \mathrm{Z}_{\mathrm{dm}} \mathrm{p}_{\mathrm{di}}+\Sigma \mathrm{Z}_{\mathrm{dm}} \mathrm{r}_{\mathrm{di}} \\
& +\mathrm{e}_{\mathrm{ijkm}},
\end{aligned}
$$

em que $\mathrm{y}_{\mathrm{ijkm}}$ é o peso corporal do indivíduo i do sexo $\mathrm{j}$, pertencente ao grupo de contemporâneos $\mathrm{k}$ (geração e eclosão), na idade em dias $\mathrm{m}(\mathrm{m}=$ $1, \ldots, 42) ; \mathrm{S}_{\mathrm{dj}}$ e $\mathrm{GC}_{\mathrm{dk}}$ são vetores que contêm coeficientes de regressão, respectivamente, para os efeitos fixos de sexo $\mathrm{j}$ e grupo de contemporâneos k; $a_{\mathrm{di}}, \mathrm{p}_{\mathrm{di}}$ e $\mathrm{r}_{\mathrm{di}}$ são os vetores dos coeficientes de regressão aleatória que descrevem a trajetória dos efeitos genético aditivo direto, de ambiente permanente do animal e dos erros de cada animal $\mathrm{i} ; \mathrm{Z}_{\mathrm{dm}}$ é o vetor dos coeficientes polinomiais de Legendre $(d=3)$ que foram utilizados para a modelagem da estrutura de covariância entre os pesos corporais na parte genética e permanente; $\mathrm{e}_{\mathrm{ijkm}}$ é o efeito aleatório residual associado a $\mathrm{y}_{\mathrm{ijkm}}$.

As análises dos pesos às várias idades foram realizadas utilizando-se o programa DFREML, opção DXMRR (Meyer, 1998a), apresentado por Silva et al. (2006). Na obtenção dos arquivos de pedigree foi utilizada a sub-rotina MTDFNRM do programa MTDFREML (Boldman et al., 1993).

De posse dos resultados das trajetórias genéticas do crescimento de cada indivíduo estimaram-se 
valores genéticos, variâncias genéticas, fenotípicas, de ambiente permanente e herdabilidades dos pesos para idades de interesse, ao longo do período de crescimento das codornas.

Para avaliar a possibilidade de modificação da curva de crescimento por meio da seleção, as equações que descreveram as trajetórias genéticas de crescimento foram agrupadas por pais (289 para EV1 e 248 para EV2) das codornas das três gerações, e os pais foram ordenados de acordo com as médias dos valores genéticos dos parâmetros b1 e b2 (declividades) e a (intercepto), por meio da sub-rotina Proc Corr (Spearman) do programa SAS (User's..., 1996), para cálculo da correlação de posto.

\section{RESULTADOS E DISCUSSÃO}

$\mathrm{Na}$ Tab. 2 são apresentadas as diversas variâncias e herdabilidades dos pesos corporais dos grupos genéticos EV1 e EV2. Os resultados mostram o comportamento diferenciado dos dois grupos genéticos: o grupo EV1, com maior variabilidade genética e valores de herdabilidade aumentando ao longo do período de crescimento (do nascimento ao $42^{\circ}$ dia de idade), enquanto o grupo genético EV2, com variabilidade genética aditiva muito baixa e maior valor estimado da herdabilidade para o peso corporal no $42^{\circ}$ dia de idade. De modo geral, as variâncias de ambiente permanente, obtidas para o grupo genético EV2, foram maiores do que as do grupo genético EV1. Deve-se registrar que o grupo genético EV2, apesar de apresentar menor variabilidade genética, o que corresponde a menor resposta ao processo seletivo, apresenta codornas com pesos corporais muito semelhantes às do grupo genético EV1.

Tabela 2. Variâncias genética aditiva e de ambiente permanente, e herdabilidades dos pesos corporais em codornas de corte dos grupos genéticos EV1 e EV2

\begin{tabular}{|c|c|c|c|c|c|c|c|}
\hline \multirow{2}{*}{ Parâmetros } & \multicolumn{3}{|r|}{ Idade } & \multicolumn{2}{|c|}{ (semanas) } & \multirow[b]{2}{*}{35} & \multirow[b]{2}{*}{42} \\
\hline & 1 & 7 & 14 & 21 & 28 & & \\
\hline \multicolumn{8}{|c|}{ EV1 } \\
\hline$\sigma_{\mathrm{a}}^{2}$ & 1,45 & 7,33 & 44,98 & 106,52 & 189,66 & 299,88 & 450,38 \\
\hline$\sigma_{c}^{2}$ & 2,36 & 16,30 & 76,70 & 139,51 & 186,66 & 230,50 & 313,81 \\
\hline$\sigma_{p}^{2}$ & 132,75 & 152,57 & 250,62 & 374,97 & 505,26 & 659,31 & 893,13 \\
\hline $\mathrm{h}_{\mathrm{a}}^{2^{\mathrm{t}}}$ & 0,01 & 0,05 & 0,18 & 0,28 & 0,38 & 0,45 & 0,50 \\
\hline \multicolumn{8}{|c|}{ EV2 } \\
\hline$\overline{\sigma_{a}^{2}}$ & 0,25 & 1,78 & 5,16 & 4,65 & 5,48 & 24,40 & 89,74 \\
\hline$\sigma_{c}^{2}$ & 3,11 & 20,74 & 108,36 & 224,04 & 352,07 & 508,41 & 740,76 \\
\hline$\sigma_{p}^{2}$ & 119,65 & 138,81 & 229,80 & 344,98 & 473,83 & 649,09 & 946,78 \\
\hline $\mathrm{h}_{\mathrm{a}}^{2^{\mathrm{t}}}$ & 0,01 & 0,01 & 0,02 & 0,01 & 0,01 & 0,04 & 0,10 \\
\hline
\end{tabular}
herdabilidade.

Os valores obtidos de herdabilidade para peso corporal do grupo genético EV1 são semelhantes aos obtidos por Akbas et al. (2004) que, ao usarem modelos de regressão aleatória para avaliarem o crescimento de codornas de corte, encontraram aumento linear nos valores de herdabilidades dos pesos corporais até o $28^{\circ}$ dia de idade, com a variância genética dos pesos corporais avaliados diminuindo ao final do período. Os valores de herdabilidades estimados do peso ao nascer, aos 35 e aos 42 dias de idade do grupo genético EV2 foram bem menores do que os observados pelos referidos autores.
Os resultados obtidos por Saatci et al. (2006) para herdabilidades de pesos corporais no $14^{\circ}$ dia de idade de codornas poedeiras, usando modelo de características múltiplas, são similares aos observados para o grupo genético EV1 $\left(\mathrm{h}^{2}=0,18\right)$ e maiores para pesos nas demais idades, exceto para peso ao nascer. Os autores (Saatci et al., 2003 e 2006) recomendam que a seleção para peso de codornas poedeiras, com melhor possibilidade de sucesso, deveria ser realizada entre o $7^{\circ}$ e o $28^{\circ}$ dia de idade. 
As moderadas a altas herdabilidades, obtidas para peso corporal do grupo genético EV1, foram menores do que as obtidas por Winter (2005) que, ao utilizar procedimentos Bayesianos, obteve estimativas de $0,25,0,43,0,53$ e 0,62 para pesos corporais aos $7^{\circ}, 14^{\circ}, 28^{\circ}$ e $42^{\circ}$ dias de idade.

As correlações genéticas, dos efeitos permanentes e fenotípicas entre pesos corporais nas diversas idades são apresentadas na Tab. 3, respectivamente, para os grupos genéticos EV1 e EV2.

Os resultados para o grupo genético EV1 mostram correlações genéticas negativas e de média a alta magnitude, entre o peso ao nascer e os demais pesos corporais, o que sugere que seleção não deve ser realizada com base no peso ao nascer. A partir do $7^{\circ}$ dia de idade, as correlações genéticas entre os pesos corporais apresentam-se altas e positivas, indicando que já se pode admitir a seleção das codornas para peso, principalmente a partir do peso corporal aos 28 dias de idade, quando ocorre aumento expressivo no valor da herdabilidade da característica, para este grupo genético.
Igualmente, pode-se verificar que, a partir do peso corporal aos 21 dias de idade, as correlações genéticas entre os pesos corporais são elevadas, o que é semelhante aos resultados obtidos por Vali et al. (2005) que, ao trabalharem com pesos corporais de duas linhagens de codornas, encontraram, no período de 35 a 63 dias, correlações genéticas positivas e altas, entre pesos corporais medidos aos $35,42,49$ e 63 dias de idade.

As correlações genéticas obtidas neste estudo são também semelhantes aos resultados observados por Winter et al. (2006) que trabalharam com pesos corporais dos $7^{\circ}, 14^{\circ}, 21^{\circ}, 28^{\circ}$ e $42^{\circ}$ dias de idade, encontrando maiores correlações genéticas entre pesos corporais no $28^{\circ}$ e $42^{\circ}$ dias de idade.

As correlações de ambiente permanente apresentam-se, de modo geral, semelhantes às correlações genéticas, o que evidencia a forte importância do efeito permanente entre os pesos corporais medidos, sendo que, de modo geral, foram maiores para o grupo genético EV2.

Tabela 3. Correlações genéticas, de efeitos permanentes e fenotípicas entre pesos corporais nas diversas idades, para os grupos genéticos EV1 e EV2

\begin{tabular}{|c|c|c|c|c|c|c|}
\hline \multirow{3}{*}{ Idades } & & & \multicolumn{4}{|c|}{ Correlações } \\
\hline & \multicolumn{2}{|c|}{ Genéticas } & \multicolumn{2}{|c|}{$\begin{array}{l}\text { Ambiente } \\
\text { permanente }\end{array}$} & \multicolumn{2}{|c|}{ Fenotípicas } \\
\hline & EV1 & EV2 & EV1 & EV2 & EV1 & EV2 \\
\hline $\mathrm{P} 1 \times \mathrm{P} 2$ & $-0,96$ & $-0,96$ & $-0,99$ & $-0,99$ & $-0,07$ & $-0,07$ \\
\hline P1xP3 & $-0,95$ & $-0,92$ & $-0,99$ & $-0,99$ & $-0,12$ & $-0,12$ \\
\hline $\mathrm{P} 1 \times \mathrm{P} 4$ & $-0,92$ & $-0,69$ & $-0,98$ & $-0,95$ & $-0,13$ & $-0,13$ \\
\hline P1xP5 & $-0,86$ & 0,20 & $-0,91$ & $-0,88$ & $-0,13$ & $-0,12$ \\
\hline P1xP6 & $-0,78$ & 0,77 & $-0,76$ & $-0,75$ & $-0,12$ & $-0,10$ \\
\hline P1xP7 & $-0,68$ & 0,90 & $-0,49$ & $-0,58$ & $-0,09$ & $-0,07$ \\
\hline $\mathrm{P} 2 \mathrm{xP} 3$ & 0,99 & 0,99 & 0,99 & 0,99 & 0,27 & 0,28 \\
\hline $\mathrm{P} 2 \times \mathrm{P} 4$ & 0,97 & 0,87 & 0,96 & 0,96 & 0,31 & 0,31 \\
\hline P2xP5 & 0,93 & 0,09 & 0,88 & 0,89 & 0,30 & 0,30 \\
\hline P2xP6 & 0,86 & $-0,56$ & 0,70 & 0,76 & 0,26 & 0,25 \\
\hline $\mathrm{P} 2 \times \mathrm{P} 7$ & 0,78 & $-0,74$ & 0,42 & 0,59 & 0,20 & 0,18 \\
\hline $\mathrm{P} 3 \times \mathrm{P} 4$ & 0,99 & 0,93 & 0,99 & 0,99 & 0,56 & 0,56 \\
\hline P3xP5 & 096 & 0,21 & 0,93 & 0,94 & 0,56 & 0,56 \\
\hline P3xP6 & 0,91 & $-0,45$ & 0,79 & 0,84 & 052 & 0,50 \\
\hline P3xP7 & 0,84 & $-0,65$ & 0,53 & 0,69 & 0,43 & 0,39 \\
\hline $\mathrm{P} 4 \times \mathrm{P} 5$ & 0,99 & 0,57 & 0,98 & 0,98 & 0,69 & 0,69 \\
\hline P4xP6 & 0,96 & $-0,08$ & 0,87 & 0,92 & 0,66 & 0,65 \\
\hline P4xP7 & 0,91 & $-0,32$ & 0,65 & 0,80 & 0,58 & 0,56 \\
\hline P5xP6 & 0,99 & 0,78 & 0,96 & 0,98 & 0,75 & 0,76 \\
\hline P5xP7 & 0,96 & 0,60 & 0,80 & 0,90 & 0,70 & 0,70 \\
\hline P6xP7 & 0,99 & 0,97 & 0,94 & 0,97 & 0,80 & 0,82 \\
\hline
\end{tabular}

$\mathrm{P} 1=$ peso corporal ao nascer; $\mathrm{P} 2=$ peso corporal aos 7 dias; $\mathrm{P} 3=$ peso corporal aos 14 dias; $\mathrm{P} 4=$ peso corporal aos 21 dias; $\mathrm{P} 5=$ peso corporal aos 28 dias; $\mathrm{P} 6=$ peso corporal aos 35 dias e $\mathrm{P} 7=$ peso corporal aos 42 dias. 
As correlações fenotípicas apresentam valores erráticos de baixa magnitude e à semelhança dos valores genéticos, apresentam-se negativas para o relacionamento entre o peso ao nascimento e os demais pesos corporais.

Os resultados observados para o grupo genético EV2, na maioria das vezes, mostram-se semelhantes aos do grupo genético EV1, quanto às três correlações (genéticas, de ambiente permanente e fenotípicas). Entretanto, para as idades referentes aos $28^{\circ}, 35^{\circ}$ e $42^{\circ}$ dias, as correlações apresentam-se erráticas, especialmente baixas e negativas, discordando de resultados de Vali et al. (2005) e Winter et al. (2006), que encontraram para pesos corporais de duas linhagens de codornas, no período de 35 a 63 dias, correlações genéticas positivas e altas.

As correlações de posto entre os parâmetros dos modelos de regressão aleatória, obtidas para os dois grupos genéticos, são apresentadas na Tab. 4.

Tabela 4. Correlações de posto entre os parâmetros das regressões aleatórias, obtidas para os dois grupos genéticos

\begin{tabular}{llll}
\hline \multicolumn{3}{c}{ EV1 } & \\
\hline & $\mathrm{a}$ & $\mathrm{b} 1$ & $\mathrm{~b} 2$ \\
$\mathrm{a}$ & & 0,97 & $-0,30$ \\
$\mathrm{~b} 1$ & & & $-0,08$ \\
\hline & EV2 & & \\
\hline & $\mathrm{a}$ & $\mathrm{b} 1$ & $\mathrm{~b} 2$ \\
$\mathrm{a}$ & & 0,83 & 0,65 \\
$\mathrm{~b} 1$ & & & 0,96 \\
\hline
\end{tabular}

Os resultados mostram novamente diferença entre os dois grupos genéticos, sendo a correlação de posto, positiva e alta para o grupo genético EV1 entre o intercepto e a declividade (a e b1) e para o grupo genético EV2 entre as declividades (b1 e b2). Entre os outros parâmetros, os valores foram negativos e baixos para EV1 e positivos e médio-alto, para o grupo genético EV2. Esta alta e positiva correlação mostra que o mesmo grupo de pais apresenta parâmetros similares, mas com grande dependência entre o intercepto (a) e declividade(b1) para o grupo genético EV1 e entre b1 e b2 para o grupo genético EV2. A ordenação dos pais se mantém ao utilizar um ou outro desses parâmetros. Os resultados mostram que existe a possibilidade de seleção visando à alteração da curva de crescimento, uma vez que os melhores indivíduos são, no processo seletivo, filhos dos melhores pais. Para o grupo genético EV1, essa seleção será mais efetiva para peso corporal, após o $7^{\circ}$ dia dentro do período de crescimento e, para o grupo genético EV2, a melhor resposta será para o peso corporal no final do período de crescimento $\left(42^{\circ}\right.$ dia de idade).

\section{CONCLUSÕES}

O grupo genético EV1 apresentou maior variabilidade genética, com estimativas maiores de herdabilidades dos pesos corporais às diversas idades, sendo os valores crescentes do nascimento ao $42^{\circ}$ dia de idade. $O$ grupo genético EV2 caracteriza-se por pouca variabilidade genética e baixa magnitude das herdabilidades dos pesos corporais, com maiores valores de herdabilidades dos pesos corporais no $35^{\circ}$ e $42^{\circ}$ dias de idade. As trajetórias genéticas de crescimento indicam que a seleção dentro do grupo genético EV1 para peso corporal poderia ser realizada com sucesso, em decorrência também dos valores de herdabilidades das características, em idades mais jovens, exceto para peso ao nascimento, enquanto, para o grupo genético EV2, a seleção poderia ser feita ao final do período de crescimento e apresentando baixa resposta à seleção.

\section{REFERÊNCIAS BIBLIOGRÁFICAS}

ALBUQUERQUE, L.G.; MEYER, K. Estimates of covariance functions for growth from birth to 630 days of age in Nelore cattle. J. Anim. Sci., v.79, p.2776-2789, 2001.

AKBAS, Y.; TAKMA, C.; YAYLAK, E. Genetic parameters for quail body weights using a random regression model. S. Afr. J. Anim. Sci., v.34, p.104-109, 2004.

BOLDMAN, K.G.; KRIESE, L.A.; Van VLECK, L.D. et al. A manual for use of $M T D F R E M L$. A set of programs to obtain estimates of variance and covariance. Lincoln: USDA/ARS, 1993. 120p.

CORRÊA, G. S. S.; SILVA, M. A.; CORRÊA, A. B. et al. Desempenho de codornas de corte EV1 alimentadas com diferentes níveis de lisina na dieta. Arq. Bras. Med. Vet. Zootec., v.59, p.1545-1553, 2007d. 
CORRÊA, G.S.S.; SILVA, M.A.; CORRÊA, A.B. et al. Exigência de metionina + cistina para codornas de corte em crescimento. Arq. Bras. Med. Vet. Zootec., v.58, p.414-420, 2006.

CORRÊA, G.S.S.; SILVA, M.A.; CORRÊA, A.B. et al. Exigência de proteína bruta e energia metabolizável em codornas de corte durante a fase de crescimento. Arq. Bras. Med. Vet. Zootec., v.59, p.488-494, 2007a.

CORRÊA, G.S.S.; SILVA, M.A.; CORRÊA, A.B. et al. Exigência de proteína bruta e energia metabolizável para codornas de corte EV1. Arq. Bras. Med. Vet. Zootec., v.59, p.797-804, 2007b.

CORRÊA, G.S.S.; SILVA, M.A.; CORRÊA, A.B. et al. Exigência de proteína bruta para codornas de corte EV1 em crescimento. Arq. Bras. Med. Vet. Zootec., v.59, p.1278-1286, 2007c.

EL FARO, L.; ALBUQUERQUE, L.G. Utilização de modelos de regressão aleatória para produção de leite no dia de controle, com diferentes estruturas de variâncias residuais. Rev. Bras. Zootec., v.32, p.1104-1113, 2003.

GRESSLER, S.L.; BERGMAN, J.A.G.; PEREIRA, A.S. et al. Estudo das associações entre perímetro escrotal e características reprodutivas de fêmeas Nelore. Rev. Bras. Zootec., v.29, p.427-437, 2000.

JOHNSTON, D.J.; BUNTER, K.L. Days to calving in Angus cattle: genetic and environmental effects, and covariances with other traits. Livest. Prod. Sci., v.45, p.13-22, 1996.

MEYER, K. "DXMRR"- a program to estimate covariance functions for longitudinal data by restricted maximum likelihood. In: WORLD CONGRESS ON GENETICS APPLIED TO LIVESTOCK PRODUCTION, 6, 1998, Armidale, Australia. Proceedings... Armidale, Australia, 1998a. p.465-466.

MEYER, K. Modeling repeated records: covariance functions and random regression models to analyze animal breeding data. In: WORLD CONGRESS ON GENETICS APPLIED TO LIVESTOCK PRODUCTION, 6,
1998, Armidale, Australia. Proceedings... Armidale, Australia, 1998b. p.517-520.

MEYER, K. Random regression to model phenotypic variation in monthly weights of Australian beef cows. Livest. Prod. Sci., v.65, p.19-38, 2000.

PEREIRA, E.; ELER, J.P.; FERRAZ, J.B.S. Correlação genética entre perímetro escrotal e algumas características reprodutivas na raça Nelore. Rev. Bras. Zootec., v.29, p.1676-1683, 2000 .

SAATCI, M.; DEWI, I.A.; AKSOY, A.R. Aplication of REML procedure to estimate the genetic parameters of weekly liveweight in oneto-on sire and dam pedigree recorded Japanese quail. J. Anim. Breed. Genet., v.120, p.23-28, 2003.

SAATCI, M.; OMED, H.; DEWI, I.A. Genetic parameters from univariate and bivariate analyses of egg and weight traits in Japanese quail. Poult. Sci., v.85, p.185-190, 2006.

SILVA, M.A.; SARMENTO, J.L.R.; TORRES, R.A.; VENTURA, R.V. Manual de utilização do programa DFREML - Máxima verossimilhança restrita livre de derivadas. 1.ed. Belo Horizonte: FEPMVZ, 2006. 99p.

USER'S guide: statistical analysis system. Release 6.11. Cary, NC: SAS Institute, 1996.

VALI, N.; EDRISS, M.A.; RAHMANI, H.R. Genetic parameters of body and some carcass traits in two quail strains. Int. J. Poult. Sci., v.4, p.296-300, 2005.

WINTER, E.M.W. Estimação de parâmetros genéticos de características de desempenho, carcaça e composição corporal de codornas para corte (Coturnix sp.). 2005. 91f. Dissertação (mestrado) - Universidade Federal do Paraná, Curitiba.

WINTER, E.M.W.; ALMEIDA, M.I.M.; OLIVEIRA, E.G. et al. Aplicação do método Bayesiano na estimação de correlações genéticas e fenotípicas de peso em codornas de corte em várias idades. Rev. Bras. Zootec., v.35, p.16841690, 2006. 